\title{
Green photonics: the role of photonics in sustainable product design
}

Berit Wessler, Ursula Tober

Berit Wessler, Ursula Tober, "Green photonics: the role of photonics in sustainable product design," Proc. SPIE 8065, SPIE Eco-Photonics 2011: Sustainable Design, Manufacturing, and Engineering Workforce Education for a Green Future, 806515 (20 April 2011); doi: 10.1117/12.882979

SPIE. Event: SPIE Eco-Photonics, 2011, Strasbourg, France 


\title{
Green Photonics - the role of photonics in sustainable product design
}

\author{
Berit Wessler, Member of the Board of Stakeholders Photonics21, OSRAM GmbH, Hellabrunner Str.1, \\ 81543 Munich, Germany
}

Ursula Tober, VDI Technologiezentrum GmbH - Photonics21 secretariat, VDI Platz 1, 40468

Duesseldorf, Germany

\begin{abstract}
Photonic technologies will play an increasingly significant role in reducing our environmental impact. In addition to the direct eco-benefits derived from the products themselves, green photonics will also impact the product design and manufacturing processes employed. Examples are discussed covering laser manufacturing, solid-state lighting, solar cells and optical communications. The importance of considering the full lifetime environmental impact of products is discussed, including raw materials, manufacture, use, and end of life issues. Industrial and legislative strategies are reviewed, and a number of specific measures are presented for accelerating the development of green photonics technologies and promoting their adoption into society.
\end{abstract}

Key words: Green photonics, energy efficiency, eco-efficiency, sustainability, laser manufacturing, solid-state lighting, LED, OLED, photovoltaic, PV, OLAE

\section{INTRODUCTION}

Photonics is the science of light and how it can be harnessed, embracing its generation, manipulation, and capture for utilisation in an enormous variety of applications, as evidenced by its increasing pervasiveness in our everyday lives. We use photonic technologies to light up our homes, offices and cities, to harvest renewable energy from the sun, to make telephone calls or surf the Internet, to enable early medical diagnosis and treatments, to establish clean and efficient manufacture of a multitude of everyday products, or to provide reliable security systems to protect us as we travel. Already, over 90 percent of all the data transferred for telecommunications is transmitted by optical fibre. In microelectronics, storage media and microprocessors are produced using optical techniques and in production engineering, laser techniques are systematically gaining ground in metrology and manufacturing. Last but not least, photovoltaic solar energy systems provide clean electricity to millions of people.

Photonics makes use of advanced technology to realize high value products and services, but its roots lie in the fundamental source of our planet's energy - the capture of light from the sun by photosynthesis in plants. This fundamental 'photonic' process is the enabler for life on our planet.

1. 'Green photonics' is the term used to encompass the application of photonics technologies that can generate or conserve energy, cut greenhouse gas emissions, reduce pollution, or yield environmentally sustainable outputs. Green photonics covers a broad range of photonic technologies and applications: photovoltaic electricity generation,

SPIE Eco-Photonics 2011: Sustainable Design, Manufacturing, and Engineering Workforce Education for a Green Future, edited by Pierre Ambs, Dan Curticapean, Claus Emmelmann, Wolfgang Knapp, Zbigniew T. Kuznicki, Patrick P. Meyrueis, Proc. of SPIE Vol. 8065, 806515 - (c) 2011 SPIE · CCC code: 0277-786X/11/\$18 - doi: 10.1117/12.882979 
highly efficient solid-state lighting (SSL), new energy-efficient communication technologies, optical sensing for improved energy efficiency \& reduced pollution, and clean manufacturing using laser processing.

If our societies are to save energy and reduce emissions of carbon dioxide, we will have to find more efficient ways to produce and consume energy, and photonics offers solutions for both. The two green photonic technologies expected to have the largest impact in the short term are solid-state light sources (light emitting diodes (LEDs) and organic LEDs (OLEDs)) for energy efficient lighting, and photovoltaic cells for generating solar electricity. Over the coming decade, the lighting domain will make a transition from the traditional technology to new digital technologies, built around LEDs \& OLEDs, photonic sensors, and built-in intelligence. Further integration with organic photovoltaic systems, all incorporated directly into the infrastructure of buildings, will contribute to the realization of energy-positive buildings and communities in the future.

Green photonics is already a key technology for improving the global balance of atmospheric carbon dioxide and will become increasingly important in the decades to come. In spite of the recent recession the overall market demand for green photonics technology is expected to achieve a 2009-2020 CAGR of $\sim 20 \%$ on average ${ }^{[i]}$. Significant drivers within the sector will be solid-state lighting and solar photovoltaics, where figures of 35\% and 30\% respectively for the 2009-2020 CAGR are predicted.

These predictions show that green photonics will be a driver for profitable growth and so further stimulate employment. Europe is leading the world in solid-state lighting and photovoltaic technologies, and their system applications. In figure 1 the European photonics production across the different sectors is shown. This indicates the fields in which Europe has particular strength.

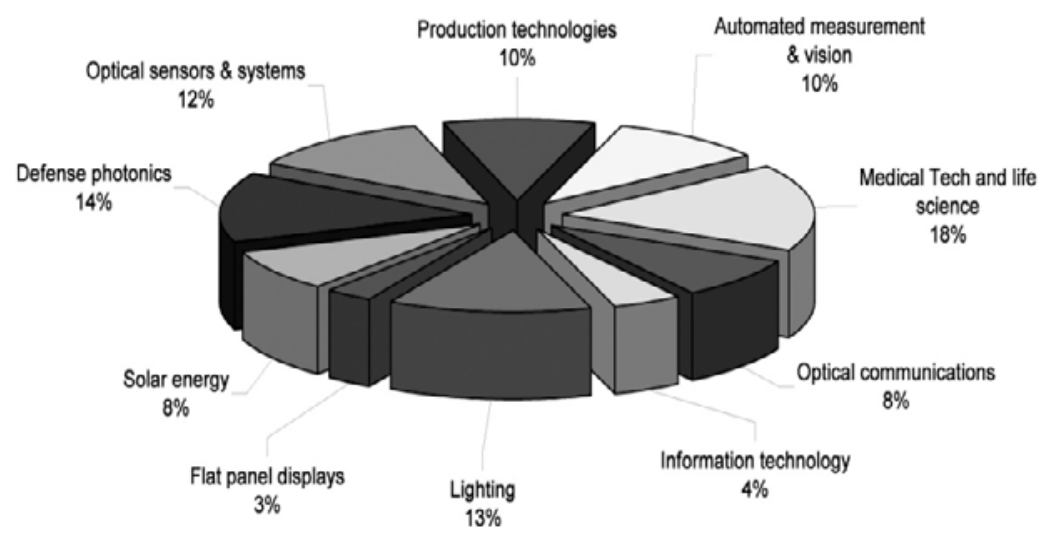

Figure 1 European photonics Production by sector [Total = 52 billion $€, 2009$ estimates] ${ }^{\text {[ii] }}$

\section{SUSTAINABILITY - WHERE AND HOW PHOTONICS CAN CONTRIBUTE}

Sustainability can be defined as ensuring that the needs of the present are met without compromising the ability of future generations to meet theirs. Thus sustainability not only includes products that have positive impact on the environment but rather comprises three categories of impact that are essential for the wealth of the society, i.e. ecological, economic and societal impact. Photonics will contribute to sustainability by offering improved environmental, economic and societal benefits (termed eco-efficiency) in three main areas; industrial design, products and production techniques. 


\section{Photonics contribution to a Sustainable Economy}

Sustainable Energy Generation

- Photovoltaic solar energy

Reduced Energy Consumption

- Solid-State Lighting (SSL)

- Communication Technologies

Enabled Eco-friendly Design \& Production

- Light-weight product design using advanced laser processing

- Material savings by precise laser cutting

- Control of production processes by sensors

- Laser processing for clean manufacturing

\section{Reduced Risk Potential}

- Sensor networks for higher safety \& security

- Environmental monitoring by sensors

\section{Eco-efficient design}

Photonics technologies are progressively being applied to manufacturing processes, most notably using light in the form of a laser, allowing processes to be handled automatically, rapidly and flexibly, producing components and products with extraordinary quality. Due to their unique properties of high power density and ultra-short pulsing, laser sources are continually revolutionizing product design as well as production technologies, and this make them a key element for sustainable economy in Europe. They provide the ideal tool for fabricating lightweight and high-strength constructions. These are already finding major applications in a wide range of applications such as crash-safe car bodies, wind turbine blades and fuel injection nozzles. The trend towards product customisation and the growing importance of industrial design, as observed most notably in consumer electronics, will benefit greatly from the flexibility, rapid reconfigurability and minimal wastage of laser-based manufacture.

Examples of the direct environmental benefits arising from new product designs enabled by a switch to laser-based manufacturing techniques are numerous and include:

- $\quad$ Laser surface treatment via thin film coating or surface modification results in increased product durability, again avoiding chemical consumption.

- Vehicles built using lightweight laser processed structures require less energy consumption in use.

- $\quad$ By using small spot sizes $(1 \mu \mathrm{m}$ instead of $10 \mu \mathrm{m})$ laser cutting \& welding techniques reduce the processed area can thus save valuable material and can realise more compact density structures. Similarly the use of lasers for localized heat treatment avoids the need for large ovens to heat the entire object.

- $\quad$ Laser processing allows new lightweight materials, such as aluminium, titanium, or even composite materials to be used instead of heavier conventional metals, such as iron and steel, with consequent improvements in strength and weight. 
- $\quad$ Laser welding and machining can be used with plastic components, avoiding the need for glues and solvents.

The key message to take from these few selected examples is the transformational increase in design flexibility offered by laser manufacturing processes to the product designers, expanding the range of materials they can use, the size \& weight of the products, and the density \& complexity of features that can now be realised.

\section{Eco-efficient products}

\section{Product Life Cycle}

When talking about energy efficiency, usually only the use-phase of a product is considered. However, to prove its overall energy efficiency, the complete life cycle of a product needs to be analysed including also raw materials, manufacturing and end-of-life treatment. This also includes consideration of issues such as abiotic depletion, human toxicity, eutrophication, acidification and global warming potentials. Life cycle assessment not only provides a way to prove the sustainability of a product, it also helps to identify potential opportunities for improvement, as it clearly indicates materials or process steps with particularly high levels of energy consumption or environmental harm. A discussion of life cycle issues for solid-state lighting is presented below.

\section{Solid State Lighting}

Currently the lighting industry worldwide faces a paradigm shift as a new era of lighting appears on the horizon: Solid State Lighting (SSL). Solid state light sources, namely LEDs and OLEDs, are mercury-free and will very soon become the light source with the highest energy efficiency. Thus, LEDs will be able to outperform all existing light sources and save significant amounts of energy, thereby not only reducing $\mathrm{CO}_{2}$ emissions, but also lowering the energy bills of consumers.

Worldwide, $19 \%$ of electricity consumption is used for lighting applications, corresponding to 2651 TWh/year ${ }^{\text {[iii] }}$ and $70 \%$ of this is being consumed by very inefficient lamps. In the Ad-hoc Advisory Group on "ICT for energy efficiency" that was established by the European Commission in 2008, the potential energy savings of LED light sources were investigated. One of the main findings reached was that about $30 \%$ of the energy for lighting could be saved by adopting more efficient lighting solutions that already exist today. However, by combining new LED solutions with intelligent light management solutions, another $30 \%$ of that energy could be saved by $2030^{[\mathrm{iv}]}$. That would imply additional savings of about $1300 \mathrm{TWh}$ and a $\mathrm{CO}_{2}$ reduction of 650 million tons, corresponding to about 2 billion barrels of oil per year. McKinsey's famous greenhouse gas abatement curves show similar results, predicting similar positive returns for the transition to efficient LED lighting systems ${ }^{[\mathrm{v}]}$.

However, energy efficiency is not all that counts. For illumination, consumers demand a high quality white light with good colour rendering and usually a warmer white appearance. Implementing this inevitably results in some reduction of energy efficiency. Nevertheless, it is essential that the lighting industry does not repeat the mistakes made with Compact Fluorescent Lamps (CFL), which even today still lack user acceptance in terms of lighting quality. Thus LED light solutions are required that offer the best compromise between efficiency and high quality white light with high colour rendering and added functionalities, such as dimming and colour tunability. Using such versatile and energy efficient lighting systems, users will be able to enhance the appearance of their homes or create an atmosphere of well being in offices, with colours that appear natural.

A life cycle assessment for LED lamps showed that less than $2 \%$ of the total amount of primary energy consumed over the lifetime is needed for the manufacturing of the LED lamp (including the energy needed for raw materials), which is almost negligible with respect to the use phase ${ }^{\text {[vi] }}$. End-of-life costs add less than $0,1 \%$ to this figure. Six environmental categories were analysed in the assessment and conclusively demonstrated that LED lamps have low impact to the environment and are even less harmful to the environment than incandescent bulbs. The total energy demand of LED lamps is equal to that of 
compact fluorescent lamps and substantially better than incandescent lamps. Future improvements of LED lamps will further cut down energy demand.

\section{Optical Fibre Networks}

Currently, the total energy required to power the Internet, including data centers, network nodes and user terminals, amounts to about 3\% of today's energy generation capacity - this is more than is used for global air traffic! With Internet traffic growing at nearly $50 \%$ each year, this demand for energy will grow relentlessly, amounting to a doubling of the required total capacity for global electricity generation in less than 10 years! Advanced photonic technologies offer significant opportunities for reducing these demands. Transmitting data as light allows it to be guided with very low losses and high capacities: a single optical fibre already carries the data equivalent of over 1000 copper cables, and the use of improved materials and novel data transport methods offer considerable further increases in data transfer. As well as transporting the data optically, new technologies also allow the data to be manipulated and processed in the optical domain, avoiding the inefficient optical-electronic-optical conversions, and so enabling switching \& digital logic functionalities with significantly reduced power consumption. More efficient photonic component designs will allow uncooled operation, avoiding the need for energy hungry temperature controllers. Major efforts are being directed towards the integration of photonic and electronic components on a single chip. In addition to the consequent dramatic reduction in device size, these visionary photonic-superintegrated circuits also offer substantial reductions in energy consumption when compared to all-electronic solutions.

\section{Organic Photovoltaics}

Organic Photovoltaics (OPV) are less mature than conventional silicon based photovoltaic materials, but offer a number of highly attractive features, not least of which is the prospect of mass production of thin-film plastic solar cells. Reel-to-reel fabrication offers large area production and substantially lowered costs, and would transform the range of deployment options for energy generation installations. To cope with the future energy demands of cities, huge areas of solar cells will be required, too large to be contemplated with conventional silicon-based PV. The big attraction of OPV technology is the potential for applying it directly in thin-film form to many urban surfaces, including windows and facades, without interfering with the existing functionalities of these building elements, thereby making it feasible to match accessible energyharvesting capacity with energy consumption needs. Since more than $40 \%$ of global energy use derives from the requirements of buildings, this technology could have a dramatic impact.

The second major application for organic photovoltaics is in the mobile devices market, where the ability to integrate a thin film, flexible energy generating layer onto the device itself or serving as a portable foil, would have major implications towards becoming less dependent on the availability of electric current.

A future vision envisaged for organic photonics will be the full integration of three functionalities; light generation (OLED), power generation (OPV) and digital processing (plastic electronics), into a single membrane. With such technology, it becomes possible to envisage readily applied membranes that provide a self-powering intelligent lighting system suitable for deployment in buildings, outdoor locations or vehicles.

\section{Eco-efficient production}

Over the coming decade photonic sensors for monitoring and controlling will play a crucial role in achieving sustainable manufacture, for which eco-friendly design and sustainable low-energy consumption production techniques will be key requirements. Compact, fully integrated sensor arrays with low-energy consumption will be able to provide real-time, 3D measurement of key process parameters, thereby allowing accurate monitoring of the full production process. The improvements in manufacturing techniques facilitated by such detailed knowledge of the process will make zero-loss production feasible, reducing economic risk and so maximizing commercial and ecological efficiency. Further opportunities for green manufacturing are offered by the use of sensors integrated with laser tools, since laser processes allow for very precise, well-controlled and therefore highly efficient energy deposition on the work piece. 
An illustrative example of the benefits offered by photonics based production is that of solar cell manufacture. Significant improvements in photovoltaic devices will be required to meet the EU Renewable Directive that targets 20\% energy supply to come from renewable sources by 2020. Laser processing is a basic prerequisite for high-volume, low-cost, non-contact manufacturing of advanced wafer-based silicon and thin-film photovoltaic cells and modules. Laser systems coupled with high-speed robotic systems linked and high performance vision systems, allow manufacturing of complex, high-efficiency 2and 3-dimensional device architectures. They also enable the use of very thin silicon wafers, so driving down the cost of silicon material. Still further device enhancements will be achieved by using advanced, high-efficiency nano-photonic structures and materials with revolutionary photonic properties, opening the way to groundbreaking new photonic components.

The role of photonic sensors and sensor networks goes significantly beyond improved manufacturing control alone, offering major improvements in the efficient use of resources. For example, the already substantial energy savings offered by solidstate lighting will be increased considerably by implementing an intelligent light management system based on distributed sensor networks and digital control that monitors usage and local conditions to optimize lighting levels. Similarly, high performance vision systems and photonic sensor networks will provide automated inspection and control, and will impact many areas of manufacturing where high throughputs are needed. Finally, photonic sensor networks provide vital monitoring of the environment in the vicinity of production equipment and materials transport systems, such as oil and gas pipelines, ensuring that any faults threatening the release of harmful or polluting materials are rapidly detected and localized.

\section{UNFORESEEN CONSEQUENCES}

One perhaps unforeseen consequence of developing technologies for lower power consumption devices is that, rather than using these to actually reduce power consumption, the improved devices or systems are instead designed to provide greater performance at the same original power consumption levels. This trend can be seen in several product sectors. For example, over the past decade display technologies have seen dramatic improvements in both quality and efficiency, yet these benefits have been manifested as larger sized displays consuming just as much or even more power than the earlier, less-efficient but smaller devices - this is seen most dramatically in flat screen TVs, where the trend is for increasingly larger screens to be the norm. Similarly in mobile phones the reduced power requirements offered by improved efficiency displays and processors are used to supply greater functionality and larger screens. Handset functionality is a more important feature to the consumer than battery life.

In LED lighting two rebound effects can be observed. First, as the energy efficiency becomes higher and higher people are less likely to switch lights off during the night anymore. Second, since LED lighting offers many new design possibilities, especially in the architectural sector, buildings are overnight illuminated is employed where no lights had been used before. So, even though is it energy-efficient, an additional source of consumption is created.

Clearly, for the full environmental benefits of eco-efficient technologies to be realised, such societal attitudes must eventually change.

\section{FUTURE STRATEGY}

It is clear that eco-efficient products \& technologies will offer huge opportunities for the European economy. Globally, it will put Europe at the forefront of providing technological solutions to the major societal challenges faced by countries across the world. This will result in improved global competitiveness, with resulting major economic benefits and the need for a highly skilled workforce within Europe. 
However, a number of hurdles must be overcome before these benefits can be realised. Most significant is probably that the initial investments in these new technologies are often higher compared to established products. Therefore eco-efficient products and technologies may not provide payback on investment until the mid- to long-term. Since shareholders and investors often apply only a quarterly or annual perspective the initial investment is consequently not made. Until full cost of ownership is used to determine procurement decisions, the short-term attraction offered by a low cost of entry product will prevail, and adoption of the new technology will be slow.

Further impediments to deployment are that the technology does not yet offer its full potential in terms of achievable performance levels, and further investment in development will be required to translate the state of the art research results in to commercial products. Finally, there is the recurrent problem faced by any new disruptive technology - how to break through the cost-volume barrier. Component prices will remain high until high volume production is rolled out, yet until low prices are possible demand will stay low.

In September 2009, the European Commission designated photonics as one of five key enabling technologies for our future prosperity. This confirmed the importance of photonics for future European economic success, and its potentially critical role in supplying solutions for the grand societal challenges now facing Europe, including the major environmental challenges. To fully exploit their critical role in tackling the environmental challenge, it will be necessary for the photonics community to act in a concerted way involving all stakeholders. This will be possible through Photonics21, the European technology platform for photonics that unites over 1700 members.

The development of new materials and device concepts for improved performance and lower costs will be pivotal for achieving the targeted penetration of eco-efficient photonic technologies. This requires a coordinated R\&D strategy to underpin this initiative, and is best achieved through collaborative research and development involving stakeholders from throughout the entire value chain. Only by working at an international level can resources be deployed in a concentrated manner, harmonizing research programs within individual countries, facilitating effective clustering of players, and avoiding fragmentation of effort. Here again, Photonics21 will have a substantial role to unite the community. Collaborative actions will also play a crucial role in exploiting photonics technologies. A number of specific measures are being planned to accelerate the development of green photonics technologies and promote their adoption into society. These include:

- Large-scale demonstration projects - these will showcase the new technologies and demonstrate the impact of next generation products in terms of costs, performance and energy saving. By fully exploiting the technical potential of photonic technologies at the system level and in a real-world environment, such projects will provide a strong validation of the approach Vital feedback will be provided on proof of the market feasibility of these new technologies, the return on investment period, and the precise potential for energy savings. Such projects could provide a valuable endorsement of the importance of these new technologies, and will play a critical role in changing the mindset of European companies and citizens.

- $\quad$ Sustainable procurement - following the outcome of large-scale project validation, the EU would then be able to set precise requirements for public procurement of green lighting for adoption across Europe. The adoption of panEuropean policies for sustainable procurement would ensure that appropriate consideration is given to full life cycle costs when selecting products and services. Though initially applied for public procurement, these policies would rapidly provide a convincing case for wider use, thereby accelerating their adoption by the market.

- $\quad$ Regulation and new business models - regulation would be extended by the introduction of certain efficiency criteria for products, thereby facilitating and encouraging the manufacture and supply of eco-efficient goods, and providing some effective protection from sub-standard imported products and solutions. New business models need to be created to maximise the benefits offered by the three sustainability pillars for wealth creation; eco-efficiency, economic growth and societal benefit. 
[i] http://www.businesswire.com/news/home/20090324006339/en/OIDA-Proposes-Criteria-Optoelectronics-CarbonEconomy-European

[ii] Photonics21: Photonics - Our vision for a key enabling technology of Europe, available online from http://www.photonics21.org/download/Events/Annual_Meeting_2011/Photonics21_Vision_Document.pdf

[iii] Light’s Labour's Lost - Policies for Energy-efficient Lighting, International Energy Agency (2006)

[iv] Ad-hoc Advisory Group, ICT and Energy Efficiency, European Commission (2008)

[v] McKinsey \& Company, Pathways to a Low-Carbon Economy - Version 2 of the Global Greenhouse Gas Abatement Cost Curve, (2009)

[vi] http://www.osram-os.com/osram_os/EN/About_Us/We_shape_the_future_of_light/Our_obligation/LED_lifecycle_assessment/OSRAM_LED_LCA_Summary_November_2009.pdf 